\title{
INVESTIGACION
}
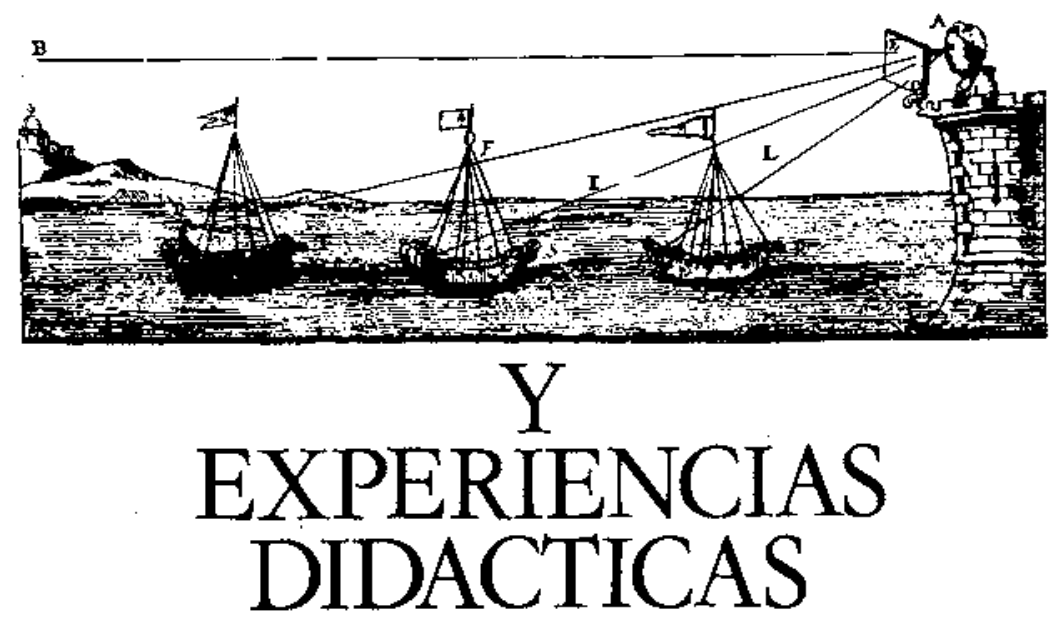

\section{PSICOLOGIA COGNOSCITIVA Y ESQUEMAS CONCEPTUALES DE LOS ALUMNOS}

DRIVER, R.

Centre for Studies in Science and Mathematics Education

University of Leeds. England.

\section{SUMMARY}

There are three main sections in this paper. The first section presents an overview of the research that has been undertaken into students' conceptions relating to mechanics. One central feature of students' (intuitive mecha* nics" is the association between their notions of force and motion. The consequences of such ideas for students' reasoning about various static and dynamic systems are described. A number of general features of students' alternative conceptions are then outlined. The second section outlines current perspectives on learning with particular emphasis being paid to the constructivist perspective; a perspective which emphasises the active role of learners in the construction of their knowledge,

The pedagogical consequences of adopting this perspective are outlined in the third section.

\section{INTRODUCCION}

Los científicos, incluidos los físicos, son imaginados

ordinariamente como "descubridores» que desvelan 
nuevos aspectos de la experiencia. Sin embargo, muchos de los más importantes desarrollos de la Física no han supuesto descubrimiento alguno, sino que han estado asociados a la reconstrucción y reformulación de las ideas usuales acerca del mundo físico; en realidad, mejor que hablar de los científicos como descubridores, habría que referirse a ellos como elaboradores de «investigaciones imaginativas».

Recientes desarrollos en el campo de la psicología cong* noscitiva, sugieren que el aprendizaje por los alumnos de materiales complejos tiene lugar mediante la organización y reestnucturación imaginativa de experiencias anteriores, más que a través de la asimilación de nueva información.

Para ilustrar hasta qué punto hacemos uso de nuestra imaginación en la interpretación del más sencilio fenómeno, consideremos que se deja caer una bola sobre una plancha metálica. Alguien que pasase en este instante veria la bola caer, golpear la plancha y rebotar hasta casi su misma altura inicial, Un físico, al interpretar el fenómeno, pensará en términos del campo gravitacional en el que la bola está situada, de la fuerza que actúa sobre ella acelerándola y haciéndole ganar energía cinética, de la deformación que tiene lugar cuando golpea la superficie metálica y, admitiendo que la colisión ha sido elástica, de la recuperación por la bola de la energía cinética, lo que permite que ascienda de nuevo. Reflexionemos un instante en la cantidad de conceptos que se hacen intervenir en este simple suceso, tales como "campo gravitatorio", «energía» e incluso un modelo de estructura de la materia. Ninguna de estas ideas es un hecho observable directamente; constituyen esquemas conceptuales intersubjetivos que se relacionan entre sí de manera precisa y que utilizamos para descubrir, predecir, y explicar sucesos como el considerado.

Un estudiante que observe el mismo suceso también aportará sus esquemas conceptuales al análisis de la situación, pero estos pueden y suelen diferir de los del físico. Precisamente, el proceso de aprendizaje está asociado a la reestructuración de estos esquemas conceptuales a partir de las nociones intuitivas iniciales.

En este artículo consideramos tres aspectos asociados a este proceso de aprendizaje en el campo de la mecánica:

1. ¿Qué conocemos acerca de los esquemas conceptuales que los alumnos utilizan para interpretar los fenómenos mecánicos?

2. ¿Qué sugiere la psicología cognoscitiva acerca de cómo se modifican los esquemas conceptuales de los alumnos?

3. ¿Qué implicaciones tiene esto para el aprendizaje?

\section{ESQUEMAS CONCEPTUALES DE LOS ES- TUDIANTES EN MECANICA}

A lo largo de la última década, el estudio de las ideas intuitivas de los alumnos acerca de los fenomenos naturales ha constituido una línea prioritaria de investigación. Estas ideas intuitivas han sido designadas de formas diversas tales como shemata (Champagne 1983), teorias ingenuas (Caramazza et Al 1981), esquemas, conceptuales alternativas (Driver y Easley 1978), (Watts 1982), o ciencia de los niños (Osborne et $\mathrm{Al}$ 1983). Profesores de física y psicólogos de distintos paises han contribuido al conocimiento de este campo y las ideas de los alumnos sobre mecánica han sido estudiadas extensamente. Una excelente y completa revisión de estos estudios ha sido preparada por McDermott (1984). En la primera parte de este trabajo resumiremos lo que puede considerarse como caracteristicas comunes más relevantes de las ideas intuitivas de los estudiantes acerca del movimiento e ilustraremos las mismas refiriéndonos a un reducido número de estudios.

\subsection{El uso de sistemas de referencia absolutos}

En un estudio del razonamiento espontáneo sobre cinética elemental de alumnos de Ensentanza Media y estudiantes universitarios, Saltiel y Malgrange (1980) prepararon cierto número de cuestiones escritas acerca de movimientos relativos, utilizando situaciones tales como un bote atravesando un rfo y gente andando o permaneciendo quieta sobre pavimentos móviles. Los autores identificaron asi entre los estudiantes una tendencia a definir el verdadero movimiento y la movilidad intrínsecamente y no con respecto a algún cuerpo o sistema de referencia específico. En otras palabras, para muchos estudiantes movimiento y reposo son substancialmente inequivalentes.

En un estudio más reciente realizado por Aguirre y Erickson (1984) sobre las concepciones de los estudiantes acerca de las caracteristicas vectoriales de la posición, el desplazamiento y la velocidad, los autores señalan que los alumnos a menudo tenian problemas para seleccionar y utilizar un sistema de referencia estandard.

La preferencia que muestran los estudiantes por un sistema de referencia absoluto no es sorprendente si tenemos en cuenta que en nuestra vida ordinaria solemos aceptar implícitamente la existencia de tales sistemas (el suelo, por ejemplo). Esta idea, obviamente, afecta a la comprensión de la Mecánica Newtoniana por los alumnos.

\subsection{El movimiento implica una fuerza}

Un amplio número de trabajos realizados con estudiantes de Enseñanza Media y Universitaria, así como con adultos no instruidos, han mostrado una tendencia muy extendida a asociar fuerza y movimiento. Esta idea está asociada a un cierto número de manifestaciones que han sido abordadas por los estudios a los que vamos a referimos. Así, Watts y Zylberztajn (1981), como parte de un trabajo realizado con un centenar de estudian- 
tes ingleses de Ensef̃anza Media de 14 años, proporcionaron un cierto número de cuestiones de respuesta múltiple, relativas a las fuerzas que actúan sobre la bala de un cañón a lo largo de su trayectoria (ver figura 1). Sus resultados mostraron que alrededor del $85 \%$ de los alumnos asociaban el movimiento a la existencia de una fuerza en la dirección del mismo.

Clement (1982) ha constatado, a través de las memorias de una práctica de laboratorio sobre el péndulo simple, que algunos estudiantes universitarios tienden a suponer la existencia de una fuerza en la dirección del movimiento de la esferita, sugiriendo, por ejemplo, que Fm (la fuerza en la dirección del movimiento) es la fuerza que hace subir al péndulo. «Si Fm no existiera, el péndulo nunca podría ascender a lo alto de su oscilación».
La simétrica de la regla según la cual el movimiento exige una fuerza es, por supuesto, si no existe fuerza no hay movimiento. McCloskey (1983) recoge una serie de investigaciones realizadas por él y sus colaboradores con estudiantes universitarios y no universitarios. En uno de dichos estudios se exploran los «conocimientos en acción» de los estudiantes pidiéndoles que suelten, mientras avanzan, una pelota que llevan en la mano, de forma que la bola dé en un blanco situado en el suelo (fig. 3). Los resultados obtenidos anotando el número de estudiantes que sueltan la pelota antes de llegar al blanco, encima del mismo, o después de pasarlo, indican que la mayoria de los estudiantes soltaron la pelota justo al pasar sobre el blanco, lo que sugiere que no tomaban en consideración la componente horizontal del movimiento de la pelota, o que asumian implícitamente que dicha componente se anula tan pronto la soltaban.

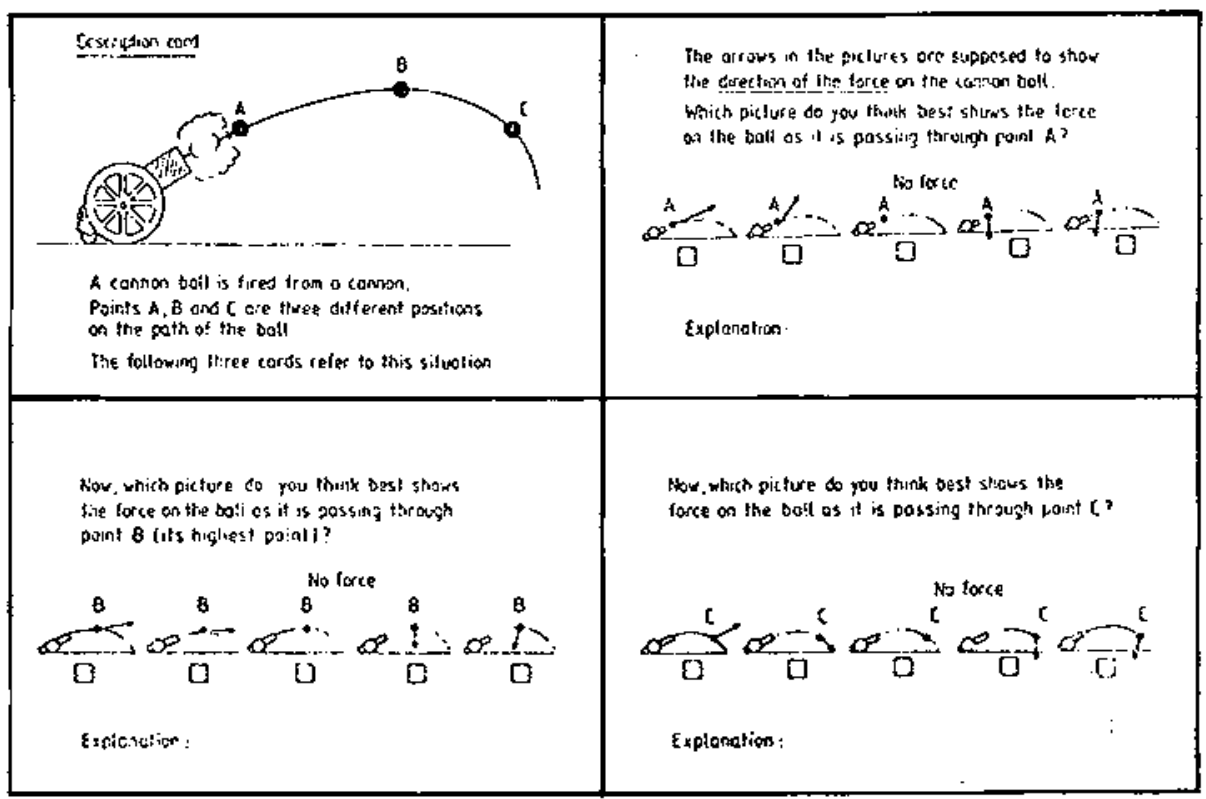

fig. I Situaciones sobre proyectiles utilizadas por Watts y Zylbersztajn

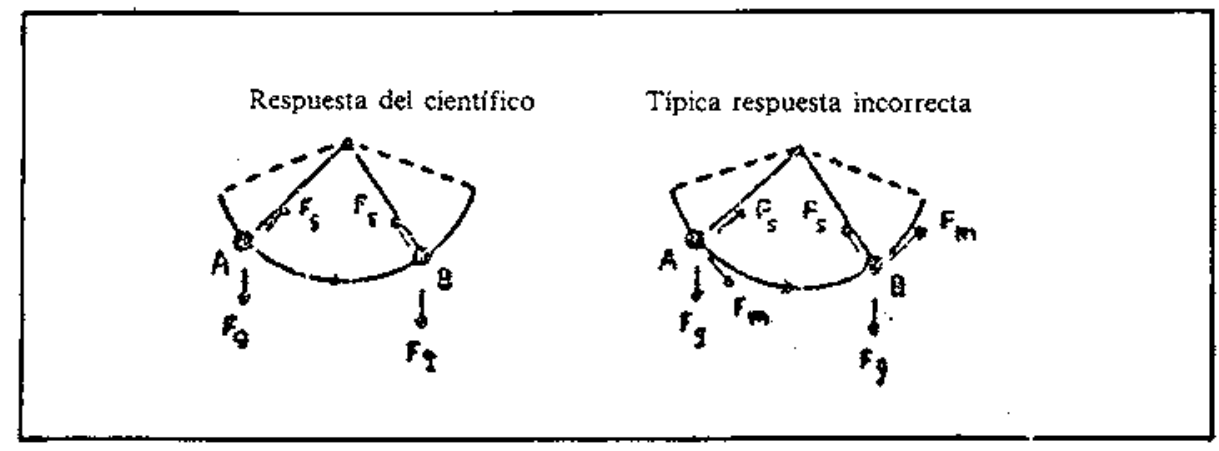

fig. 2 Fuerzas sobre un pénduto en movimiento (Clement 1982). 


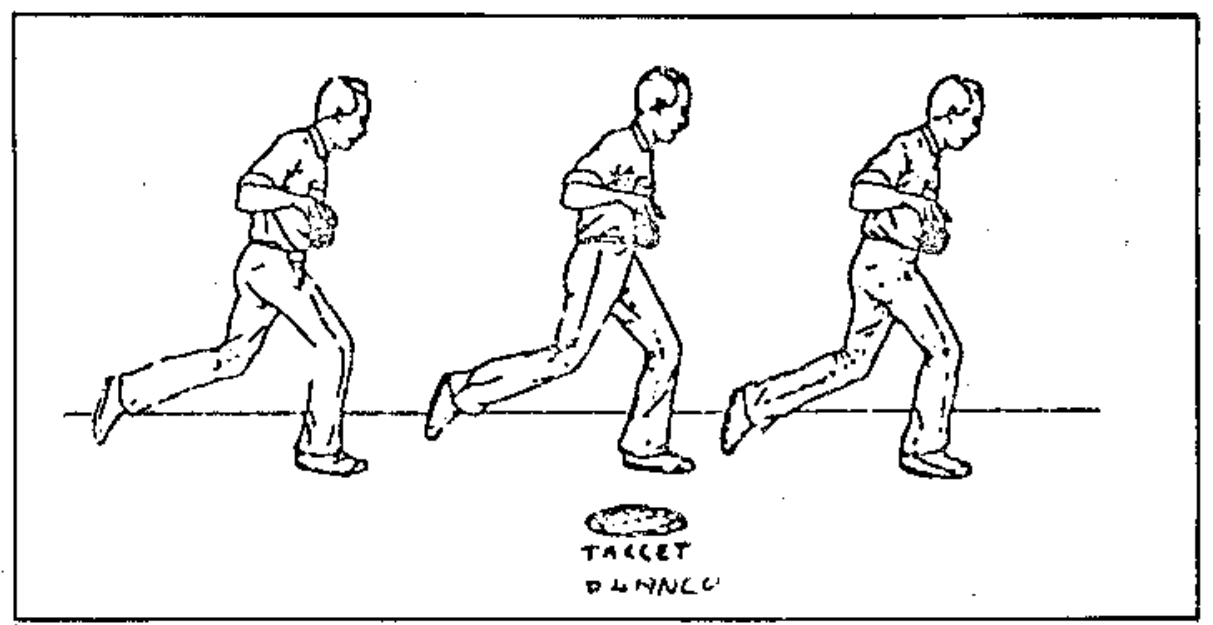

fig. 3 Predicción de la trayectoria de un objeto que cae (McCloskey 1983).

\subsection{Se precisa que una fuerza actúe continuamente pa- ra mantener un movimiento}

La idea de que para mantener un movimiento continuo se precisa que una fuerza esté continuamente actuando, forma parte del esquema «movimiento implica fuerza». Durante una investigación que he realizado con jóvenes alumnos de Enseñanza Media que estudiaban Mecánica (Driver 1983), al preguntarle a una niña - que estaba describiendo lo que sucede cuando pelotas de distinta masa ruedan por tierra- por qué las pelotas se paraban, contestó: «No lo sé. ¿Por qué se paran?. Bueno, siempre se paran. Después de impulsarlas avanzan tanto como el impulso... lo fuerte que fuera y cuando se gasta vuelve a como estaba antes».

Las observaciones ordinarias nos dicen que los objetos disminuyen de velocidad y se paran después que los hemos empujado o lanzado, y esto se interpreta comúnmente suponiendo que se ha gastado la fuerza que se les comunicó. McDermott (1984) describe que cuando se preguntaba a estudiantes universitarios que comunicaran a un disco, situado sobre un banco de aire, un movimiento rectilíneo y uniforme utilizando chorros de aire, sus primeros intentos consistían a menudo en aplicar una continua serie de ráfagas.

\subsection{La fuerza varía con la rapidez del movimiento}

En un conocido trabajo, Viennot (1979) propuso a estudiantes franceses, belgas e ingleses, de Enseñanza Media y Universidad, un cierto número de cuestiones escritas, una de las cuales representaba seis bolas de malabarista, todas a la misma altura del suelo pero en diferentes posiciones de sus respectivas trayectorias (ver figura 4). Se preguntaba a los alumnos si las fuerzas que actuaban sobre todas las bolas eran o no idénticas en el instante representado. Los resultados indicaron que, incluso a nivel universitario, los estudiantes asociaban fuerza con velocidad y sugerían por ejemplo: «Las velocidades son diferentes, luego las fuerzas lo serán también».

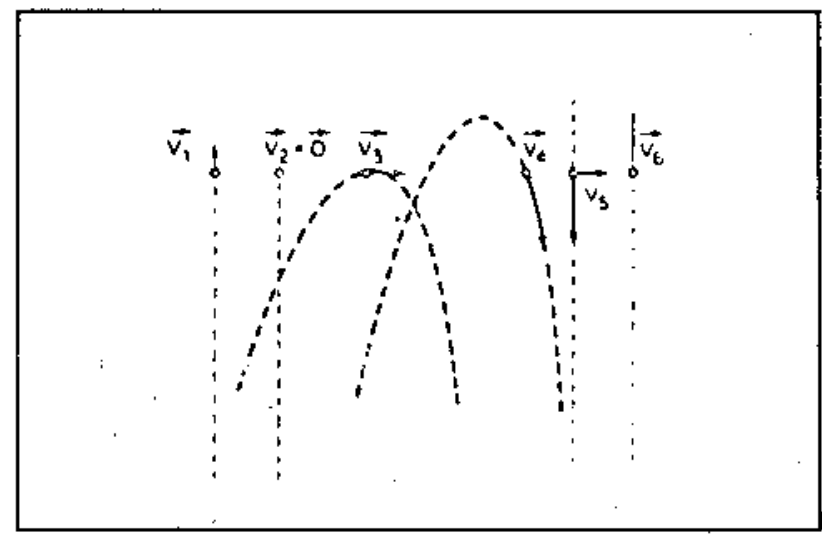

fig. 4 Predecir las fuerzas que actúan sobre pelotas en el aire (Viennot, 1979).

En su artículo Viennot distingue entre dos ideas de fuerzas utilizadas por los estudiantes y hace la importante observación de que las distintas ideas son utilizadas en diferentes contextos, resaltando las caracteristicas de las situaciones en las que cada tipo de idea es manejado:

Una de las tareas utilizada por Clement (1982) ilustra también este punto: se muestra a estudiantes de ingeniería una moneda lanzada verticalmente hacia arriba y se les pide que dibujen una o más flechas que indiquen la dirección de cada una de las fuerzas que actúa sobre la moneda cuando alcanza el punto más alto. Al final del curso, el $72 \%$ de los estudiantes dieron respuestas incorrectas. Una respuesta típica incluia dos 
fuerzas, una hacia arriba, procedente de la mano $(\mathrm{Fm})$ y otra hacia abajo $(\mathrm{Fg})$, con razonamiento como: «mientras la moneda asciende, la fuerza de la mano impulsa a la moneda y ha de ser mayor que $\mathrm{Fg}$, pues en caso contrario descenderiay.

\subsection{El movimiento tiene hugar en la dirección de la fuerza aplicada}

En los trabajos hasta aquí reseñados, aparecía implícitamente la idea de que ha de actuar una fuerza en la dirección del movimiento. Igualmente se ha identificado la idea simétrica de que el movimiento tiene lugar en la dirección de la fuerza aplicada.

Di Sessa (1982), describe un estudio sobre las interacciones de estudiantes con un programa de ordenador denominado "dinatortuga». Un objeto sobre la pantalla, la "dinatortuga», obedece a la $I^{2}$ y $2^{a}$ leyes de Newton, de forma que permanece en reposo o se mueve en línea recta con rapidez constante cuando no actúa ninguna fuerza sobre ella y se le puede comunicar impulsos en cualquier dirección. Al pedir a los estudiantes que envien a la tortuga hacia un blanco, muy frecuentemente le comunican un impulso dirigido en línea recta hacia el blanco, sin tener en cuenta su movimiento inicial. Este comportamiento sugiere que los estudiantes esperan que el objeto se mueva en la dirección de la fuerza aplicada, ignorando su cantidad de movimiento inicial.

\subsection{Un objeto en reposo no puede ejercer fuerza}

Muchos estudiantes tienen dificultades en torno a las fuerzas que se ejercen en una situación de equilibrio estático. Parece subyacer la idea de que ha de estar clara la capacidad de moverse de un objeto para aceptar que ejerce una fuerza. Durante unas observaciones que realizé en una clase (Driver 1973), se produjo una discusión entre dos niños de 12 años, Richy y Tím, acerca de las fuerzas que actuaban sobre un objeto que colgaba en reposo de un soporte rígido. Richy argumentaba que sobre el cuerpo actuaba una fuerza vertical hacia arriba debido al soporte, además de una fuerza vertical hacia abajo. Su companero Tim se manifestaba en contra arguyendo que el soporte se limitaba a sostenerlo. Richy, entonces, soltó el objeto del soporte y lo sostuvo en su mano, desarrollándose el siguiente diálogo:

$R$. Bien. ¿Qué pasa ahora? Voy a soltarlo del soporte y sostenerlo en la mano.

$T$. Tienes que ejercer una fuerza hacia arriba con tu mano, porque si no lo hicieras se caería al suelo.

$R$. Cierto! El soporte ha tenido que hacer una fuerza hacia arriba también.

$T$. No actúa del mismo modo. Este (el soporte) solo está manteniéndolo en su posición.

$R$. ¿Pero eso mismo hace mi mano!

$T$. No, tu mano no lo mantiene perfectamente inmó- vil. Le está levantando. ¿Cómo podria esto (el soporte) levantar algó? No está vivo.

La capacidad de la mano para moverse parecia esencial en la argumentación de Tim, haciéndole dif́cil aceptar la lógica de la argumentación de Richy.

Un razonamiento similar ha sido observado por Minstrell (1982) con estudiantes de últimos cursos de Ensenanza Media y por Clement y Brown (1983) con estudiantes universitarios. También se han investigado las ideas de los estudiantes acerca de las tensiones que actúan sobre cables y muelles en diversos montajes. Así, por ejemplo, Watts y Zylbersztajn (1981) sețalan que en una competición de tiro de cuerda, los estudiantes argumentan que la fuerza que ejerce el ganador es mayor que la ejercida por el perdedor. Por mi parte he constatado que la situación representada en la fig. 5 provoca discusiones abundantes incluso entre licenciados en ciencias físicas. Algunos argumentan que la lectura del dinamómetro será nula porque las fuerzas se anulan mutuamente. Otros dicen que ambos pesos «tiran el uno contra el otro" y que, por tanto, se suman sus efectos.

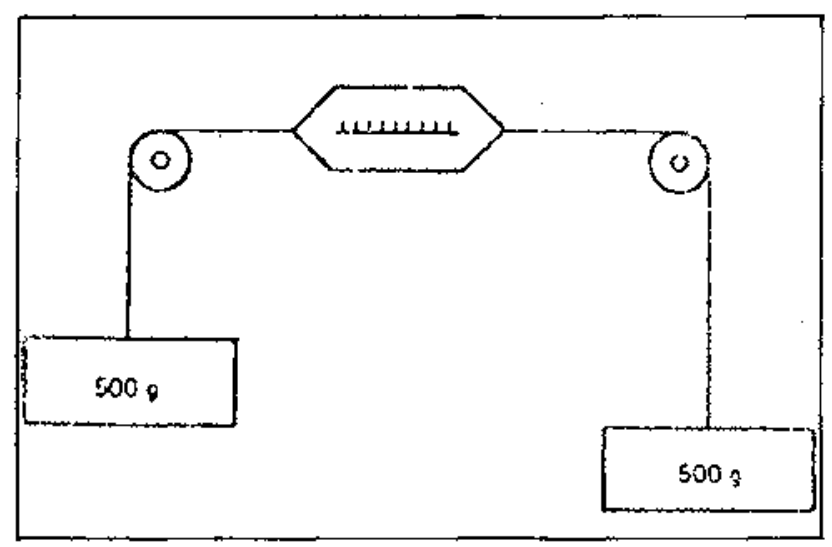

fig. 5 Predicción de la tensión de un resorte. (Driver 1983).

\subsection{Ideas acerca de la gravedad}

Existe cierto número de ideas relacionadas entre sí que han sido identificadas en las concepciones de los estudiantes sobre la gravedad.

En algunos casos los estudiantes consideran que la fuerza de la gravedad es mayor cuanto más arriba se encuentra el objeto. Así, un niño de 11 años me explicaba mientras sostenía una bolita:

"Cuanto más alto llegue mayor será el efecto de la gravedad sobre la misma, porque si usted se queda ahi y alguien deja caer un guijarro sobre él (sic), sólo le dará una punzada. Pero si yo la dejo caer desde un aeroplano, se aceleraria más y más rápido y cuando golpeara a alguien le mataría” (Driver 1983). 
Con relación a las nociones de fuerza y movimiento, la idea đe que los cuerpos más pesados caen más aprisa que los ligeros, persiste incluso entre estudiantes universitarios (Gunstone y White 1981) (Watts 1982). Por otra parte, algunos alumnos asocian la gravedad con el aire y la atmósfera, de forma que al pedirles que predigan la trayectoria de un proyectil lanzado en un recipiente en el que se ha hecho el vacio, un elevado número de estudiantes noruegos de Enseñanza Media y Universidad afirmaban que la trayectoria serfa una línea recta horizontal, arguyendo que «la gravedad necesita un medion (Sjoberg y Lie 1981).

\subsection{Algunas características generales de los esquemas conceptuales alternativos de los estudiantes}

Terminaremos este apartado del artículo con algunas observaciones generales acerca de las características de los esquemas conceptuales de los alumnos:

\section{$i$ Se trata de esquemas dotados de cierta coherencia} interna.

En muchos casos, los argumentos utilizados por los estudiantes para explicar sus concepciones (como son la existencia de una fuerza en la dirección del movimiento, etc) son válidos en determinados contextos. En general no se trata de ideas irracionales, sino simplemente fundamentadas en premisas diferentes de las de la mecánica Newtoniana. Responden a intentos racionales de explicar las experiencias físicas.

En la vida cotidiana, donde, por ejemplo, la fricción está siempre presente, no aparece la necesidad de construir una mecánica que tenga en cuenta los movimientos sin rozamiento.

ii Los estudiantes manejan un lenguaje impreciso y términos indiferenciados para expresar sus ideas. En algunos casos pueden incluso no hacerlas explicitas para ellos mismos.

iii Ideas intuitivas similares son detectadas en estudiantes de diferentes medios y edades.

Puesto que vivimos en un medio con las mismas características físicas, tal vez no sea sorprendente que los esquemas conceptuales que construimos para interpretar nuestras experiencias sean similares e, incidentalmente, muestran algunas semejanzas con ideas aparecidas a lo largo de la historia de las ciencias.

iv Los esquemas conceptuales alternativos son persistentes y no se modifican fácilmente mediante la ensertanza tradicional.

La frecuencia con la que los estudiantes universitarios de Física hacen uso de, por ejemplo, la asociación fuer$\mathrm{za} /$ movimiento, es un índice de la persistencia de dichas ideas. Así, al analizar cómo los estudiantes universitarios resuelven ciertos problemas incluidos habitualmente en textos de Física, se constata cómo la asociación intuitiva entre fuerza y movimiento aparece en, por ejemplo, los diagramas de fuerza que dibujan (Heller y Hungate 1984).

Cierto número de investigadores ha señalado que los estudiantes pueden aplicar correctamente las ecuaciones Newtonianas en los problemas usuales $\mathrm{y}$, sin embargo, fracasan cuando se enfrentan a fenómenos físicos reales. Ello sugiere que una de las razones de la persistencia de dichas ideas es que no se ha producido la integración del conocimiento que los estudiantes tenían del mundo físico con el conocimiento que proporcionan las relaciones algébraicas abstractas.

$v$ Los esquemas conceptuales de los alumnos no son simples construcciones ad hoc.

¿Puede decirse que los diferentes aspectos de las ideas intuitivas de los alumnos acerca del movimiento constituyen una teoría coherente?. Clement (1983) sostiene que las ideas intuitivas no son simples reglas ad hoc, sino que representan una teoria coherente que, atendiendo al paralelismo histórico, denomina «teoria del impetus». Sugiere, además, que es esa coherencia de la teorfa del impetus lo que contribuye a su estabilidad. Sin embargo, aunque pueda aceptarse la coherencia de la teoría, sabemos que en la práctica los estudiantes no son consistentes en su uso, como han mostrado Viennot y otros.

Una clara muestra de ello ha sido presentada por White (1983) a partir de una serié de cuestiones planteadas en entrevistas realizadas a 40 alumnos que habian seguido un curso PSSC de Física. Las cuestiones planteadas se referian todas al desplazamiento en el espacio exterior de una nave espacial que disponia de un cohete capaz de proporcionarle impulsos discontinuos idénticos. Cuando se preguntaba a los estudiantes qué ocurriría si se daba un único impulso a la nave, el $80 \%$ predecía correctamente la producción de un movimiento $\sin$ fin de rapidez constante. Y el $60 \%$ predecia también correctamente el movimiento resultante de comunicar a la nave tres impulsos sucesivos en ángulos rectos. Sin embargo, en otras dos cuestiones planteadas más tarde a los mismos estudiantes se les preguntaba qué harian a) para que la nave siguiera una trayectoria circular y b) para que siguiera una trayectoria cuadrada. Las respuestas, recogidas en la figura 6 , indican que sólo un porcentaje notablemente inferior tenía en cuenta, al abordar estas cuestiones, que el movimiento resultante de un objeto depende no sólo del impulso que se le comunica, sino también de la cantidad de movimiento inicial. Esta inconsistencia plantea una cuestión importante desde el punto de vista educativo: ¿Cuáles son los aspectos de un determinado contexto que conducen al uso de las ideas intuitivas? ¿Cuáles son las ideas que se utilizan en cada contexto?

yi No se pueden atribuir todas las dificultades de los estudiantes a sus esquemas conceptuales alternativos. 


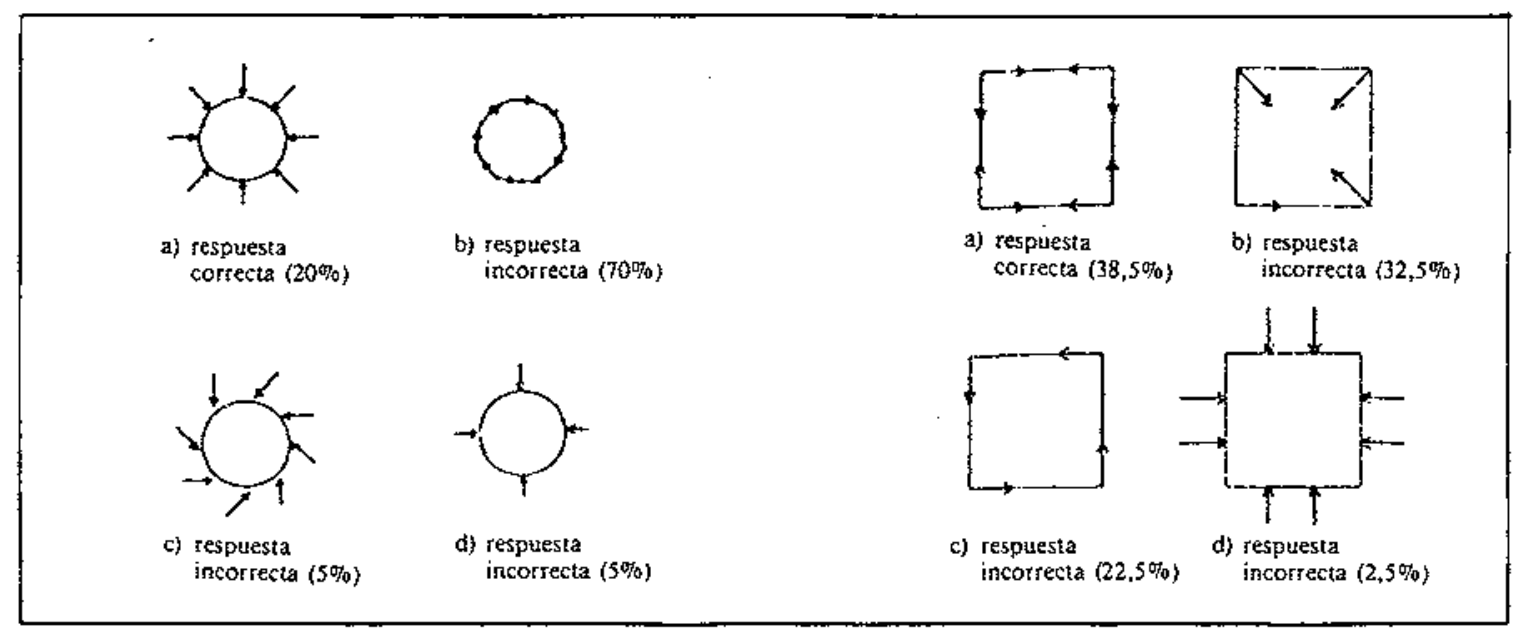

fig. 6 Predicción del movimiento de una nave espacial propulsada por impulso de un cohete (White, 1983).

Tanto Trowbridge y McDermott (1981) como White (1983) llaman la atención contra la suposición de que todas las dificultades que los alumnos tienen para comprender la mecánica Newtoniana sean debidas a la existencia de potentes ideas intuitivas. Estas investigaciones han identificado otras fuentes de dificultad en el uso del lenguaje formal de la ciencia, como por ejemplo gráficos y notación vectorial.

Hasta aquí hemos revisado lo que conocemos sobre esquemas conceptuales de los alumnos acerca del movimiento mecánico. Nos ocuparemos a continuación de cómo dichos esquemas pueden modificarse, es decir, de cómo puede tener lugar el aprendizaje.

\section{CONCEPCIONES ACTUALES SOBRE EL APRENDIZAJE}

\subsection{Tres visiones de cómo tiene lugar el aprendizaje}

Como educadores, nuestro interés sobre las concepciones de los alumnos han de ir más allá de la catalogación de las ideas alternativas o intuitivas: no sólo nos interesan las ideas que los estudiantes poseen ya al iniciar el aprendizaje, sino también la dinámica del cambio cognoscitivo, para que esta pueda guiar así nuestra enseñanza y la planificación del curriculum.

Hasta bastante recientemente el pensamiento de los profesores de ciencias y muchas de las recomendaciones de tipo pedagógico han estado dominadas por dos grandes concepciones sobre el aprendizaje: la orientación evolutiva y la orientación behaviorista (Driver 1982).

La tradición evolutiva, que ha estado presidida por el trabajo de Piaget y colaboradores, postula la existencia de estructuras cognoscitivas en el cerebro que procesan la información e influencian las interacciones del individuo con el medio externo. Se considera que esas estructuras se desarrollan en etapas, correspondiendo la etapa de pensamiento abstracto o formal - cuyas características son esenciales para la comprensión de la ciencia- al período de la adolescencia. De acuerdo con esa visión, las dificultades experimentadas por los alumnos en la comprensión de los conceptos físicos han sido atribuidas a la falta de estructuras lógicas adecuadas en los alumnos. Las implicaciones educativas de esta teoría han sido, o bien intentar adecuar los materiales de un curso a la etapa del desarrollo cognoscitivo del alumno (Shayer y Adey 1981), o bien diseñar. programas para acelerar el proceso de maduración. Los resultados de varias investigaciones realizadas por psicólogos y educadores muestran que las estructuras lógicas que utilizan los estudiantes dependen en gran medida del contexto de la tarea, poniendo así en cuestión la idea de etapas coherentes.

La segunda concepción que ha ejercido una influencia notable ha sido la behaviorista. Esta teoria no hace suposiciones sobre la organización interna del conocimiento en quien aprende, ni postula ninguna limitación de edad para el aprendizaje. Adopta una visión esencialmente optimista del aprendizaje, postulando que pueden construirse series de comportamientos, habilidades o conceptos de complejidad creciente, a través de programas de instrucción cuidadosamente elaborados. Estos programas son diseñados mediante un detenido análisis lógico del material a enseñar. La persistencia de los esquemas conceptuales alternativos de los estudiantes da una idea de las dificultades inherentes a esta orientación.

Recientemente ha comenzado a emerger una tercera visión del aprendizaje. Resnick (1983) describe así esta concepción: «Una aportación clave de los trabajos de psicología cognoscitiva de los últimos años ha sido que el comportamiento inteligente de una persona no depende de unos "procesos de pensamiento" abstractos, sino que depende íntimamente de la clase de conocimiento que la persona tiene acerca de la situación par- 
ticular en cuestión... para poder comprender cómo tiene lugar un aprendizaje complejo, es preciso estudiar cómo la gente aprende materias particulares. Por esta razón, hoy hay psicólogos cognoscitivos estudiando matemáticas en particular, o física en particular, etc, etc. Esta visión đe cómo tiene lugar el aprendizaje está siendo desarrollada por psicólogos cognoscitivos, expertos en inteligencia artificial, especialistas en lenguaje y profesores de ciencias y de matemáticas. Algunos describirían esta concepción como basada en el proceso de información. Yo prefiero denominarla visión constructivista, porque así se subraya el papel esencialmente activo de quien aprendes.

Presentaré aquí una interpretación personal de las características esenciales de esta orientación constructivista, aprovechando de forma ecléctica el trabajo de un cierto número de autores.

\subsection{Principales características de Ja visión construc- tivista}

i Lo que hay en el cerebro del que va a aprender tiene importancia.

Esta concepción sugiere que todos construimos representaciones del saber que utilizamos para interpretar la experiencia. Los resultados del aprendizaje no sólo dependen de la situación de aprendizaje y de las experiencias que proporcionamos a nuestros estudiantes, sino también de los conocimientos previos de los mismos, de sus concepciones y motivaciones. Algunos ejemplos discutidos en la primera parte de este artícuIo ilustran que estas concepciones influyen de maneras diversas en la forma en que los estudiantes interaccionan cont los materiales de aprendizaje: No sólo influyen en sus interpretaciones de los fenómenos, las explicaciones que dan de los mismos, sino que determinan la dirección de su observación, focalizan su atención y orientan los experimentos que realizan.

ii Encontrar sentido supone establecer relaciones.

Los conocimientos que pueden conservarse largo tiempo en la memoria no son hechos aislados, sino aquelios muy estructurados e interrelacionados de múltiples formas. Como físicos podemos apreciar las propiedades de una estructura altamente organizada de conocimientos; podemos reconocer las relaciones exactas representadas por un mapa conceptual de los aspectos de la mecánica (Karplus 1981).

Esto, sin embargo, representa únicamente la estructura axiomática de parte de la materia, sin señalar conexiones con las muchas y variadas instancias del mundo físico. Las investigaciones sobre las concepciones intuitivas de los alumnos sugieren que quizás todos tenemos una serie de ideas y conexiones semejantes que derivan de la experiencia física. Asf, cuando vemos aproximarse un balón hacia nosotros nos preparamos para recibir su sacudida al cogerlo; Ianzamos objetos con alguna expectativa de la trayectoria que seguirán; tomamos en consideración el peso de un objeto antes de empujarlo por el suelo. Este conocimiento físico está también estructurado, pero como no to hacemos explicito, no siempre lo relacionamos con el conocimiento axiomático; de hecho podemos incluso no ser conscientes de las suposiciones que hacemos.

Actualmente está emergiendo la comprensión de otro aspecto importante de cómo se organiza el conocimiento humano: se ha realizado la distinción entre lo que se ha denominado conocimiento declarativo (conocer «qué») y conocimiento procesal (conocer «como»). Estudios recientes sugieren que el razonamiento humano se describe mejor mediante representaciones de procedimientos.

Rumelhart y Norman (1981) sugieren que «en muchos sentidos parece que las personas poseen características más próximas de las que se atribuyen a los sistemas procesales que de las atribuidas a los sistemas declarativos. Nuestra habilidad para razonar y utilizar nuestro conocimiento parece depender fuertemente del contexto en el que el conocimiento es adquirido. La mayor parte de los razonamientos que hacemos, aparentemente no suponen la aplicación de habilidades de razonamiento de carácter general. Más bien parece que la mayor parte de nuestra capacidad de razonamiento está asociada a cuerpos particulares de conocimiento.

Un tercer punto en conexión con los sistemas de estructuración del conocimiento humano es la comprensión de que nuestras emociones juegan un papel fundamental en la orientación de nuestras funciones cognoscitivas, por lo que deben ser tenidas también en cuenta al considerar el aprendizaje (Norman 1981).

iii Quien aprende construye activamente significados.

Estudios sobre las formas en que comprendemos un texto (Wittrock 1980) o comprendemos el mundo físi$\mathrm{co}$, sugieren que interpretamos activamente nuevas experiencias mediante analogías, a partir de las estructuras de conocimientos que ya poseemos, lo que a su vez puede modificar dichas estructuras. Ello significa que lo que determina nuestra actividad en cualquier situación, no es tanto lo que extraemos de ella cuanto las construcciones que aportamos a la misma. Esta propiedad constnuctiva del cerebro ha sido claramente demostrada por los fenómenos de ilusión óptica como el incluido en la figura 7 (Gregory 1983). La perspectiva constructivista sugiere que más que "extraer» conocimiento de la realidad, la realidad sólo existe en la medida que la construimos.

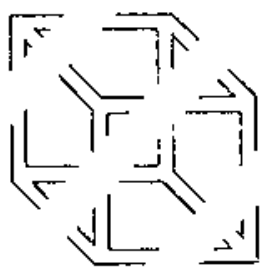

fig, 7 Ilusion 6ptica. 
También sabemos que la construcción de significados, ya sea a partir de un texto, de un diálogo o de una experiencia fisica, implica un proceso activo de formulación de hipótesis o realización de ensayos, que son contrastados mediante experiencias sensoriales. Si hay acuerdo decimos que "comprendemos»; en caso contrario intentamos con nuevas construcciones o abandonamos la situación como "carente de sentido». Este proceso ha sido documentado por Clement (1983) en un estudio de cómo los expertos utilizan analogias cuando intentan resolver problemas de dinámica. También Di Sessa (1983) describe las explicaciones que estudiantes universitarios dan de sencillos fenómenos físicos y muestra el papel central que juegan las analogias en el desarrollo de su pensamiento.

En algunas situaciones las construcciones ya existentes son utilizadas para encontrar el sentido de las experiencias sin que sean necesarios grandes cambios en la estructura conceptual del sujeto. En otras, el acto de dar sentido implica un proceso analógico durante el cual las ideas existentes son utilizadas de una nueva forma, conduciendo posiblemente a una nueva construcción. Este proceso de cambio en la organización del conocimiento o reestructuración (Rumelhart y Norma 1981) presenta interés en la enseñanza de la Física, dado que es este tipo de aprendizaje el que - de acuerdo con los resultados analizados en la primera parte de este artículo- sería necesario que se produjera en los alumnos. Es preciso, sin embargo, dejar claro que los cambios en la estructura conceptual no tienen ligar fácilmente.

En un importante articulo, Posner, Strike, Hewson y Gertzog (1982) postulan las condiciones necesarias para que tenga lugar el cambio conceptual. A partir de los escritos de Toulmin (1972) sobre filosofía de la ciencia, identifican cuatro condiciones!

1. Es preciso que se produca insatisfacción con los conceptos existentes.

2. Ha de existir una nueva concepción mínimamente inteligible que

3. debe ser inicialmente plausible y

4. ha de ser potencialmente fructífera, abriendo nuevas áreas de investigación.

De esta concepción activa de construcción de significado se derivan dos puntos. El primero es que la comprensión implica la existencia de expectativas y no ser simplemente un receptor pasivo de información. El segundo concierne a la problemática cuestión de lo que se acepta como aprendizaje significativo. Desde el punto de vista constructivista, aceptamos como significativo cuando hay acuerdo entre nuestras experiencias y nuestras concepciones. Esto tiene consecuencias para la enser̃anza y el aprendizaje, sugiriendo un desplazamiento desde la preocupación por la validez objetiva de las actividades o respuestas de los alumnos a una actitud de ayudar a los alumnos a organizar sus pro- pias experiencias en una forma que sea coherente para ellos.

iv Los estudiantes son responsables de su propio aprendizaje.

No se trata de una afirmación utópica; desde la perspectiva constructivista constituye el reconocimiento de una condición necesaria del aprendizaje.

Los estudiantes son responsables de su aprendizaje en el sentido de que han de dirigir su atención hacia la tarea de aprendizaje y hacer uso de sus propios conocimientos para construir ellos mismos el significado en la situación de aprendizaje, incluso cuando tienen una actitud exteriormente pasiva (como cuando están leyendo o escuchando). Desgraciadamente muchas situaciones de aprendizaje no estimulan a los estudiantes a encontrar el sentido de lo que están experimentando. EI pensamiento de los estudiantes puede ser cortocircuitado fácilmente con la búsqueda de la «respuesta correcta» al problema del libro de texto o de las «lecturas correctasy en el trabajo de laboratorio.

Esta sección del artículo ha sintetizado las caracteristicas principales de la visión constructivista del aprendizaje. Ahora consideramos cuáles son las implicaciones pedagógicas de esta perspectiva.

\section{LAS IMPLICACIONES PEDAGOGICAS DE LA VISION CONSTRUCTIVISTA DEL APRENDIZAJE}

Existen dos orientaciones básicas usualmente adoptadas en la investigación de los progresos en el aprendizaje de los estudiantes de física. A una la denominamos orientación de instrucción directa y a la segunda orientacion de cambio conceptual.

\subsection{Orientación de instrucción directa}

Los estudios realizados con esta orientación se han centrado en las formas de mejorar los resultados obtenidos por los alumnos en la resolución de los ejercicios de los libros de texto. Comparando las actuaciones de "expertos» y "novicios» en resolver problemas (Larkin y Reif 1979) (Simon y Simon 1978) se han indentificado cierto número de importantes diferencias entre ambos.

Los expertos tienden a realizar un análisis cualitativo inicial del problema, construyendo una representación significativa del mismo en términos de conceptos físicos. Así, por ejemplo, tienden a dibujar diagramas que no representan únicamente la situación física, sino que incluyen relaciones entre magnitudes físicas que pueden no ser mencionadas explfcitamente en el enunciado (como dibujar diagramas vectoriales de las fuerzas). Los novicios por otra parte, tienden a omitir este proceso y se dirigen directamente hacia las manipulaciones algebraicas de las magnitudes dadas en el enuncia- 
do. Se ha constatado, además, que cuando dibujan diagramas, estos evidencian concepciones intuitivas o alternativas de las fuerzas.

Algunas investigaciones didácticas han mostrado que las orientaciones que mejoran la actuación de los principiantes incluyen su entrenamiento especifico en la descripción del problema en términos de ideas físicas antes de utilizar ninguna expresión matemática y en especificar las relaciones estructurales entre magnitudes utilizando analogias explicitamente ensertadas (Larkin 1979) (Riley 1983). Heller y Reif (1984) se han referido a un sistema altamente estructurado de preparación para la resolución de problemas en el campo de la mecánica con el que se ha mostrado que, si los estudiantes siguen las etapas prescritas en el modelo, generan «buenas» descripciones de los problemas y ello mejora notablemente su éxito en la obtención de una solución correcta. Esta orientación se está extendiendo actualmente a un sistema de ensentanza con ayuda de ordenadores (Hellen y Hungate 1984). Los autores identifican el fundamento de esta orientación como «desarrollo gradual de conocimientos controlado por expertos». Inicialmente el principiante observa, mientras el tutor modela la actividad a realizar; gradualmente el aprendiz toma la responsabilidad de más y más decisiones, hasta que finalmente acomete la resolución de un problema completo sin intervención del tutor. Esta aproximación que proporciona un «andamiaje tutorials, supone que el control del conocimiento es interiorizado progresivamente por el estudiante como resultado del proceso.

Estas orientaciones no toman en consideración los conocimientos y representaciones que el alumno posee, sino que intentan construir esquemas alternativos a través de programas cuidadosamente estructurados.

La investigación realizada en esta línea tiende a concebir el conocimiento físico como axiomático y no problemático y a no poner de relieve la relación entre los conceptos y los fenómenos físicos. Considera al aprendiz como estando inicialmente bajo control externo y tiende a resaltar los procesos analíticos en la resolución de problemas.

Los resultados obtenidos con los estudios iniciales de este tipo parecen prometedores, pero cabe esperar a ver hasta qué punto los estudiantes generalizan los conocimientos adquiridos al utilizar esta orientación en otros contextos, en particular en situaciones físicas.

\subsection{La orientación de cambio conceptual}

En contraste con la orientación precedente, un planteamiento alternativo consiste en tomar en consideración explicitamente las concepciones iniciales de los estudiantes y diseñar formas de cambiarlas. En mi opinión, los estudios emprendidos con esta perspectiva contemplan el conocimiento físico como más problemático y experimental, tendiendo a dar más énfasis a las experiencias de los estudiantes sobre fenomenos fi- sicos y considerando que el aprendizaje se regula internamente y que la resolución de problemas implica más un razonamiento holistico, analógico, que analítico.

Se ha iniciado un cierto número de estudios en los que se ha implementado y evaluado una orientación del aprendizaje con cambio conceptual. Se considera generalmente que esta orientación implica un cierto número de pasos:

\section{i La identificación y clarificación de las ideas que ya} poseen los alumnos.

Como técnicas para implementar esto se ha utilizado la enseñanza socrática (Champgne, Gunstone i Klopfer 1982) (McDermott, Piternick y Rosenquist 1980), la discusión entre los estudiantes (Gilbert y Pope 1982), la resolución de problemas en pequeños grupos (Whimbey y Lochhead 1980) y la observación/discusión basada en el trabajo de laboratorio (Lochhead 1983).

ii La puesta en cuestión de las ideas de los estudiantes a través del uso de contraejemplos.

La efectividad de demostraciones individuales o con pe queños grupos para conseguir esto ha sido documen. tada por diversos autores, entre los que se encuentran Stavy y Berkovitz (1980) y Rowell y Dawson (1983). Pero aunque esta orientación parece haber dado buenos resultados en algunos casos, varios autores se han referido a la resistencia de los estudiantes a modificar sus concepciones. Los contraejemplos parecen poder debilitar la creencia de los estudiantes en sus ideas, pero sin proporcionar nada a cambio.

\section{iii Invención o introducción de conceptos.}

Se introducen nuevas ideas bien mediante brainstorming entre los alumnos (Nussbaum y Novick 1981), bien mediante presentación explícita del profesor o a través de los materiales de instrucción. Aquí resulta importante el uso de metáforas para posibilitar que los estudiantes relacionen sus conocimientos previos y las nuevas construcciones y desarrollar una representación descriptiva antes de que una teoría sea presentada en forma axiomática. Se sugiere, además, que se presen. ten las teorias de forma que los estudiantes las contemplen como tentativas que posiblemente requieran modificaciones.

\section{iv Proporcionar oportunidades a los estudiantes pa- ra usar las nuevas ideas.}

Varios estudios han sugerido la importancia de dar a los estudiantes oportunidades para usar las nuevas ideas de varias formas y hacer así que adquieran confianza en las mismas (Cosgrove, Osborne y Tasker 1982). Una forma interesante y efectiva de proporcionar a los estudiantes experiencia en el campo de la mecánica es a través de la simulación con microordenadores (Di Ses* sa 1982) (White 1983) (Hewson 1983). Mediante ejercicios de control de la trayectoria sobre la pantalla de 
un objeto programado para obedecer la física Newtoniana, se ha conseguido modificar las ideas intuitivas de los alumnos sobre fuerza y movimiento. Cierto número de factores pueden contribuir a la efectividad de esta estrategia de intervención:

La situacion presentada en el microordenador está gobernada por reglas claras, mientras que los fenómenos físicos son más confusos; además, los estudiantes pueden obtener retroalimentación inmediata sobre la efectividad de sus hipótesis.

Por otra parte, cabe pensar en la conveniencia de introducir modificaciones en los problemas tradicionales, dando ocasión para analizar y resolver problemas en los que se de exceso de información o en los que los mismos estudiantes hayan de decidir cuales son las magnitudes que se precisan (Gil y Mtnez-Torregrosa 1983), con lo que se impulsa a los alumnos a considerar la situación física superando la habitual manipulación abstracta de las relaciones algebraicas.

También algunas actividades prácticas diseñadas para mostrar la utilidad de los principios físicos (en situaciones como el funcionamiento de los cinturones de seguridad, el diseño de bicicletas o la dinámica de los deportes) pueden contribuir a que los jóvenes vean la relevancia y utilidad de lo que de otro modo les aparece como ideas abstractas que no merece la pena «embarcar» en su pensamiento".

\section{RESUMEN Y CONCLUSIONES}

En conclusión sugiero que nuestra actual comprensión de la forma en que se produce el aprendizaje tiene importantes y signiticativas implicaciones en la enseñanza y en el mismo aprendizaje. Ello requiere un cambio en la forma de enseñanza, pasando de la mera «transmisión» de conocimientos a una orientación en la que los estudiantes son impulsados a construir sus propios significados. Las estrategias utilizadas a tal efecto, en un determinado contexto temático, incluyen los siguientes pasos:

$i$ La identificación de las ideas previas de los alumnos.

ii La puesta en cuestión de las mismas, si necesario, mediante el uso de contraejemplos.

iii La invención o introducción de conceptos.

iv $\mathrm{El}$ uso de las nuevas ideas en un amplio abanico de situaciones.

Estas son estrategias de enseñanza que pueden utilizarse en el desarrollo de un curso. Además hay que considerar cierto número de implicaciones más a largo plazo para el disento del curriculum.

1. La necesidad de un planteamiento «de larga duración» del curriculum de Física: no se puede pensar en que los individuos modifiquen en una o dos ho- ras ideas que han desarrollado a lo largo de su existencia.

2. La consideración del curriculum en una óptica de desarrollo.

$\mathrm{Al}$ analizar las concepciones de los estudiantes estamos en la comprensión de identificar los bloques más conflictivos y podemos utilizar este conocimiento en la preparación de actividades que sean útiles para reestruc * turación del pensamiento de los estudiantes.

Una de las dificultades con que tropiezan los alumnos en la comprensión de, por ejemplo, las fuerzas estáticas, es que conceptualizan los objetos sólidos como ab. solutamente rígidos. Cuando se muestra que los sólidos se deforman, los estudiantes de Enseñanza Media aceptan más fácilmente que pueden ejercer una fuerza.

Conviene también que consideremos seriamente la necesidad de un curriculum que no suponga que los alumnos comprenden una teoria en su forma más elabora. da desde la primera vez que se les enseña. (De hecho, si somos honestos, no podemos pretender que nosotros seamos capaces de ello). Digamos, más explícitamente, que nuestra estrategia es el desarrollo progresi. vo de las concepciones de los alumnos sobre los fenómenos físicos a lo largo de un período de cinco a diez años y no conseguir un dominio de conceptos en un mes.

\section{Proporcionar al inicio de un tema un amplio abani- co de experiencias físicas}

Ello introduce una nueva fundamentación para el tra. bajo de laboratorio; no se trata de entrenar a los estu* diantes en el uso del material de laboratorio ni tampoco llevarles a realizar investigaciones abiertas (aunque ambas cosas sean útiles). En vez de ello se trataria de suministrar opurtunidades para que los alumnos se «enreden" con un fenómeno y su pensamiento se acomode con su experiencia. Esto puede implicar trabajo cualitativo de laboratorio durante el cual los estudiantes observen, recojan y representen sus observaciones de diversas formas y discutan sus interpretaciones de dichos sucesos.

Destrás de estas recomendaciones subyace no sólo una perspectiva de cómo tiene lugar el aprendizaje, sino también una visión de para qué enseñamos física.

Algunas estrategias de enseñanza pueden ser apropiadas si estamos interesados en of recer un entrenamiento en la realización de un tipo particular de ejercicios. Pero si contemplamos la física como un vehículo para una educación más general y para el desarrollo de èstudiantes de física más autónomos, nuestros objetivos pueden ser considerablemente más ámplios. En este caso podemos intentar desarrollar en los alumnos:

i Una comprensión que integre el conocimiento fi: sico y el teórico. 
ii El interés por plantear cuestiones y la confianza para aplicar los conocimientos en situaciones novedosas.

iii La voluntad de someter a análisis y de valorar el propio pensamiento en vez de apoyarse en autoridades externas.

$i v$ La toma de conciencia de los principios que orien- tan el propio pensamiento y de que estos pueden precisar cambios; y al propio tiempo saber apreciar que otra gente pueda tener diferentes principios.

$v$ Imaginación y voluntad de experimentación, apertura a nuevas comprensiones, a las ideas de otra gente y al propio crecimiento personal.

\section{REFERENCIAS BIBLIOGRAFICAS}

AGUIRRE, J., ERICKSON, G., 1984, Students' conceptions about the vector characteristics of three physics concepts. Journal of Res in Sci. Teaching 21 (5), 439.457.

CARAMAZZA, A., MCCLOSKEY, M., GREEN, B., 1981, Naive beliefs in «sophisticated subjecte; misconceptions about trajecctories of objects. Cognition 9, 117-123.

CHAMPAGNE, A., GUNSTONE, R., KLOPFER, L., 1983, Effecting changes in cognitive structurs amongst physics students. Paper presented at the symposium on Stability and the American Association, Montreal.

CLEMENT, J., 1982, Student preconceptions in introductory mechanics. Am. J. of Physics 50 (1), 66-71.

CLEMENT, İ., 1982, Student alternative conceptions in mechanics: a coherent system of reconceptions. In Helm $\mathrm{H}$. and Novak J. (Eds.) Proceedings of the International Siminar 'Misconceptions in Science and Mathematics' Ithaca N.Y. Cornetl University.

CLEMENT, J., BROWN, D., 1983, Using analogical reasoning to deal with 'deep' misconceptions in physics. Working paper, University of Massachusetts, Amherst M.A.
COSGROVE, M., OSBORNE, R., TASKER, R., 1982, TOward generative learning. Working paper No: 205. Science Education Research Unit, University of Waikato, New Zealand.

DRIVER, R., 1973, The Representation of Conceptual Frameworks in young adolescent science students. Unpub. $\mathrm{Ph}$. D. thesis, Univ. of Illinois, Urbana, Illinois.

DRIVER, R., 1982, Children's Learning in' Science. Educational Analysis 4 (2), 69-79.

DRIVER, R., EASLEY J., 1978, Pupils and paradigms: A Review of literature related to concept Development in Adolescent Science Students. Studies in Science Educa. tion 5, 61-84.

DI SESSA, A., 1982, Unlearning Aristotelian physics: A study of knowledge-based learning. Cognitive Science 6 , 37-75.

DI SESSA, A., 1983, Phenomenology and the evolution of intuition. In Mental Models Eds. D. Gentner and A. Stevens, Lawrence Erlbaum Associates. 
GIL PEREZ, D., MARTINEZ-TORREGROSA, J., 1983. A model for problem-solving in accordance with scientific methodology, European Journal of Science Education, 5 (4), 447-455.

GILBERT, J., POPE, M., 1982, School children discussing energy. Report of Institute of Educational Development, University of Surrey.

GREGORY, R., 1983, Visual perception and illusions. In Miller J. States of Mind. British Broadcasting Corporation: London.

GUNSTONE, R., WHITE, R., 1981, Understanding of Gravity. Science Ed. 65 (3), 291-299.

HELLER, J.I., REIF, F., 1984, Prescribing effective human problem-solving processes: Problem description in physics. Cognition and Instruction, 1.

HELLER, J.I., HUNGATE, H.N., 1984, Theory-based instruction in description of mechanical problems. Paper presented a AERA meeting, New Orleans, April 1984.

HEWSON, P., 1983, Microcomputers and conceptual change: the use of a microcomputer program to diagnose and remediate an alternative conception of speed. Paper presented at AERA meeting, Montreal, 1983.

KARPLUS, R., 1981, Educational aspects of the structure of physics. Am. J. Phys, 49 (3), 238-241.

LARKIN, J.H., 1979, Processing information for effective problem solving. Engineering Education, December 1979, 285-288.

LARKIN, J.H., REIF, F., 1979, Understanding and teaching problem-solving in physics. Eur. J. Sci. Educ. 1 (2).

LOCHHEAD, J., 1983, Beyond Emile. Paper presented at the AERA meeting, Montreal, 1983.

MCCLOSKEY, M., 1983, Intuitive physics. Scientific American 248, 122-130.

MCDERMOTT, L.C., 1984, An overview of research on conceptual understanding in mechanics. Physics Today, 37, 7,24 .

MCDERMOTT, L., PITERNICK, L., ROSENQUIST, M., 1980 , Helping minority students succeed in science I, II and III. Journal of College Science Teaching. Jan, March, May.

MINSTRELL, J., 1982, Explaining the 'At rest' conditions of an object. Physics Teacher 20310.

NORMAN, D.A., 1981, Perspectives on cognitive science Lawrence Erlbaum Associates, Hillsdale.

NUSSBAUM, J., NOVICK, S., 1981, Brainstorming in the Classroom to invent a model: a case study. School Science Review 62, No. 221, 771-778.

OSBORNE, R.J. BELL, B.F., GILBERT, J.K., 1983 Science Teaching and Children's views of the world. European Journal of Science Education 5 (1), 1-14.
POSNER, G.J., STRIKE, K.A., HEWSON, P.W., GERTZOG, W.A., 1982, Accommodation of a scientific conception: toward a theory of conceptual change. Science Education, 66 (2), 211-227.

RESNICK, L.B., 1983, Mathematics and science learning: A new conception. Science, 220, 477-478.

RILEY, M., 1983, Instructional methods that make a difference: structural understanding and the acquisition of problemsolving skill. Paper presented at AERA meeting, April 1983, Montreal.

ROWELL, J.A., DAWSON, C.J., 1983, Laboratory counter examples and the growth of understanding in science. European Journal of Science Education 5 (2), 203-216.

RUMELHART, D.E. NORMAN, D.A., 1981, Analogical processes in learning. In J.R. Anderson (Ed). Cognitive skills and their acquisition, Lawrence Erlbaum Associates, Hillsdale.

SALTIEL, E., MALGRANGE, J.C., 1980, 'Spontaneous' ways of reasoning in elementary kinematics. Eur. Phys. $1,73-8$.

SHAYER, M. ADEY, P., 1981, Towards a Science of Science Teaching, Heinemann.

SIMON, D.P. SIMON, H.A., 1978, Individual differences in solving physics problems. In R. Siegler (Ed.) Children's thinking: What develops? Erlbaum, Hillsdale N.J.

SJOBERG, S., LIE S., 1981, Idees about force and movement among Norwegian pupils and students. Report 81-11. Institute of Physics Report Series, Universtiy of Oslo.

STAVY, R., BERKOVITZ, B., 1980, Cognitive conflict as a basis for teaching quantitive aspects of the concept of temperature. Science Education, 64 (5), 679-692.

TOULMIN, S., 1972, Human Understanding Vol. I The Co llective Use and Evolution of Concept Princeton University Press.

TROWBRIDGE, D.E., MCDERMOTT, L.C., 1981, Investigation of student understanding of the concept of acceleration in one dimension. American Journal of Physics 49, 242 .

VIENNOT, L., 1979, Spontaneous reasoning in elementary dynamics. Eur. J. Sci. Educ. I (2), 205-222.

WATTS, D.M., 1982, Gravity don't take it for granted. Phys. Educ. 17, 116-121.

WATTS, D.M., ZYLBERSZTAJN, A., 1981, A survey of some ideas about force. Physics Education 16, 360-365.

WHIMBEY, A., LOCHHEAD, J., 1980, Problem solving and comprehension Franklin Institute Press: Philadelphia.

WHITE, B., 1983, Sources of difficulty in understanding Newtonian dynamics. Cognitive Science 7, 41.

WITTROCK, M.C., 1980, Learning and the brain. In Wittrock, M.C. (Ed.) The brain and psychology. Academic Press: New York. 